\title{
Facteurs de résilience chez des orphelins rescapés du génocide qui vivent seuls dans les ménages au Rwanda (Association Tubeho)
}

\author{
Berthe Kayitesi, Rollande Deslandes et Christine Lebel \\ Département des sciences de l'éducation, Université du Québec à Trois-Rivières
}

\begin{abstract}
RÉSUMÉ
Cet article relate les principaux résultats d'une recherche effectuée auprès d'orphelins rescapés du génocide des Tutsis du Rwanda qui sont devenus responsables d'eux-mêmes après 1994. Le but de cette étude était de comprendre les facteurs de protection qui ont permis à certaines et certains d'entre eux de survivre dans leurs ménages et en même temps de poursuivre leurs études jusqu'à l'université malgré de multiples facteurs de risque auxquels ils ont été confrontés durant et après le génocide. Ces facteurs de protection sont associés à des caractéristiques individuelles, familiales et communautaires. L'article présente brièvement le contexte de l'étude, la méthode utilisée et les résultats obtenus suivis des recommandations quant à l'orientation de recherches ultérieures dans le domaine de la résilience et de la promotion de celle-ci dans la communauté rwandaise et dans des contextes similaires.
\end{abstract}

Le génocide des Tutsis du Rwanda qui a eu lieu en 1994 a laissé de grandes séquelles, dont le traumatisme aigu des rescapés et rescapées du génocide. Ce traumatisme est le résultat des expériences vécues pendant le génocide, du vide et de la coupure existentielle causés par l'absence des leurs et du processus de deuil que la tragédie rend long et difficile. En plus de cet impact psychologique, les survivants et survivantes évoluent dans un contexte socio-économique caractérisé par la pauvreté qui les accule à la misère et qui rend la plupart d'entre eux incapables d'envisager l'avenir avec optimisme (Bazigaba, 2005).

Cet article a été possible grâce à une bourse d'études accordée à la première auteure par l'Agence canadienne de développement international (ACDI) et à une aide financière obtenue des fonds de recherche des professeures Deslandes (directrice du mémoire) et Lebel (co-directrice). Faire parvenir toute demande d'information concernant cet article à: Berthe Kayitesi,kaybe78@yahoo.fr, bkayi072@uOttawa.ca 
De plusieurs catégories des rescapés et rescapées du génocide des Tutsis du Rwanda, celle des orphelins (féminins et masculins) chefs de ménages nous interpelle dans cette étude. Gishoma (2005), qui s'est penché sur le traumatisme psychique vécu par cette population, mentionne que ces derniers vivent une expérience traumatique double, liée d'une part à la destruction collective en tant que tel, et d'autre part au fait que malgré leurs blessures, ils ne peuvent, en grande partie, compter que sur euxmêmes pour survivre. À cause de cette situation, la majorité d'entre eux ont abandonné les études; d'autres décrochent à défaut de trouver du matériel scolaire, d'obtenir un soutien affectif et surtout de concilier leurs nouvelles responsabilités familiales avec leurs études (Gishoma, 2005). Cependant, une minorité de cette population arrive à s'en sortir et à poursuivre leurs études. Voilà la raison pour laquelle cet article traite de la résilience auprès de ceux et celles qui, malgré cette nouvelle identité d'orphelins chefs de ménages, ont pu cheminer normalement dans leurs études. La scolarisation dans le cadre de cycles supérieurs est prise en compte parce que beaucoup de jeunes adultes qui composent les ménages d'orphelins au Rwanda étaient sur les bancs de l'école en 1994, et aussi, parce qu'avoir pu continuer leurs études semble un des éléments favorables à leur processus de résilience.

\section{CADRE DE RÉFÉRENCE}

Très tôt en 1994, le nombre d'orphelins du génocide s'estimait à 95000 selon l'enquête de l'UNICEF (2004). Certains de ces orphelins ont été placés dans des familles d'accueil ou des orphelinats et d'autres se sont retrouvés seuls dans des ménages d'orphelins. Dans ces ménages où ils sont livrés à eux-mêmes, le plus âgé ou le plus responsable doit assurer la subsistance du reste de la famille, sans pour autant avoir la maturité nécessaire, ni disposer des ressources suffisantes. Cette situation des ménages d'orphelins était rare dans le Rwanda d'avant 1994, car à la mort des parents il y avait presque toujours un membre de la parenté (grands-parents, oncles, tantes, etc.) pour prendre la relève et servir de substituts parentaux (Dushime, 2004). Or, lors du génocide, les familles entières ont été décimées, et beaucoup d'orphelins se sont retrouvés seuls au monde, sans parenté adulte pouvant s'en occuper.

L'Association de coopération et de recherche pour le développement (ACORD) (2001) souligne l'impact de cette nouvelle situation issue du génocide en ces termes: «C'est la nature et l'ampleur, ainsi que la manière de gérer la situation des orphelins qui vivent seuls dans les ménages qui ont changé suite aux conséquences de la guerre, des massacres et du génocide sur la famille et la société rwandaise » (p. 15). Dans une perspective de prise en charge, des efforts ont été déployés par le gouvernement rwandais et les associations des rescapés et rescapées du génocide. À titre d'exemple, le Fonds national d'assistance aux rescapés du génocide (FARG) vient en aide aux rescapés et rescapées en matière de santé, de scolarité et parfois de logement. Bien que cette chance soit offerte sur le plan scolaire, les conditions de la vie ordinaire empêchent certains orphelins à en profiter.

Sur le plan psychologique, par exemple, la reviviscence des expériences vécues en 1994 occupe une place prépondérante au sein des ménages d'orphelins et limite leur projection dans l'avenir (Gishoma, 2005). L'urgence de la survie et la combinaison des études et des responsabilités familiales ont conditionné l'abandon scolaire de beaucoup d'orphelins rescapés du génocide (Bazigaba, 2005; Gishoma, 2005; Mukuzanyana, 2004; Muragizi Kanama, 2004). Deux jeunes chefs de ménage de 


\section{FACTEURS DE RÉSILIENCE CHEZ DES ORPHELINS RESCAPÉS DU GÉNOCIDE}

Nyamata interviewés par Gishoma (2005) soulignent les raisons de leur décrochage scolaire en ces termes: "C'était clair que nous devrions tous arrêter d'étudier et travailler. [...] J'ai retiré les deux autres enfants de l'école pour m'aider à cultiver » (p. 36).

Le poids de cette situation fait également écho dans ce discours de Charles dans l'étude de Bazigaba (2005): "Même un parent planifie l'intervalle entre les enfants qu'il met au monde. Cela rend facile leur éducation. Mais lorsqu'on trouve cinq enfants d'un coup, il est difficile de gérer cette situation. » (p. 59). Ce poids qui pèse sur la vie des orphelins chefs de ménages s'accentue par la pauvreté dans laquelle ils baignent (Bazigaba, 2005; Dushime, 2004; Gishoma, 2005; Mukuzanyana 2004; Muragizi Kanama, 2004; Rwabidadi, 2000). Un autre facteur à prendre en considération est le fait que ces orphelins chefs de ménage qui assument des responsabilités parentales ont perdu leur identité de frère ou de sœur qui est pourtant réelle en assumant des rôles parentaux, et n'ont pas celle de parent qu'ils essaient de représenter (Bazigaba, 2005).

Malgré ces conditions de vie alarmantes, certains orphelins luttent pour s'en sortir, ne décrochent pas et arrivent à combiner la vie dans les ménages et leur scolarité. D'où l'intérêt à identifier les facteurs qui contribuent à leur processus de résilience. La résilience permet de constater que, face à des situations à risque élevé, l'être humain peut s'en sortir, grâce à l'interaction de différents facteurs présents chez lui et dans son entourage (Cyrulnik, 2003; Poletti \& Dobbs, 2001; Vanistandael \& Lecomte, 2000). Les événements qui présentent un risque pour l'individu sont connus sous le terme de facteurs de risque, tandis que les éléments susceptibles d'atténuer l'impact de ces facteurs de risque sont connus comme étant des facteurs de protection (Anaut, 2003; St-Onge, 2003). Lorsqu'il est question des facteurs présents chez l'individu, Cyrulnik (2003) parle des ressources internes qui sont imprégnées au cours du développement par une figure d'attachement, un objet hypersignifiant, comme l'objet maternel quelle que soit la figure d'attachement: mère, père, autre femme, autre homme, etc. Poletti et Dobbs (2001) abondent dans le même sens et précisent que les ressources de l'individu viennent de «son patrimoine génétique, des circonstances de sa petite enfance, des messages qu'il a reçus, des attachements qu'il a pu créer et de la sécurité qu'il a ressentie dans ses attachements, de sa manière d'entrer en contact avec les adultes » (p. 35). Ainsi, la qualité de la relation qui s'établit entre l'enfant et les adultes de son entourage détermine ses chances de devenir résilient lorsqu'il sera confronté à l'adversité (Benard, 2004; Vanistendael \& Lecomte, 2000).

Mis à part ces ressources acquises lors de la petite enfance, la résilience résulte de la signification que la communauté ou la société donne au traumatisme et des ressources dont ces groupes disposent pour aider le «blessé à s'en sortir » (Ehrensaft \& Tousignant, 2001). Pour les enfants blessés, le milieu scolaire est l'un des endroits susceptibles de contribuer à la construction de la résilience et ce, grâce aux liens qui s'établissent entre eux, avec leurs enseignants et enseignantes ou encore avec d'autres adultes du milieu scolaire (Anaut, 2003; Benard, 2004; Bouteyre, 2004; Poletti \& Dobbs, 2001; Royal, 2001). Notons que le lien et le sens sont deux facteurs essentiels dans le processus de résilience, comme le souligne Lecomte (2004) à l'aide de nombreux exemples. 


\section{QUESTION DE RECHERCHE}

La question générale de cette recherche sur la résilience des orphelins qui vivent seuls dans les ménages se lit comme suit: À quoi les étudiantes et étudiants rescapés du génocide qui vivent seuls dans les ménages attribuent-ils leur résilience? De cette question découle une question spécifique: Dans une perspective écosystémique, quels sont les facteurs de protection qui ont aidé les étudiantes et étudiants rescapés du génocide qui vivent seuls dans les ménages à cheminer jusqu'à l'université?

\section{MÉTHODE}

Pour répondre à la question de recherche, nous avons privilégié une démarche qualitative de type exploratoire. Cette démarche a permis de dégager les facteurs de protection qui ont permis aux orphelins rescapés du génocide qui vivent seuls dans les ménages au Rwanda d'avancer dans leur scolarité malgré les différents facteurs de risque auxquels ils ont dû faire face ou auxquels ils font encore face.

\section{Participants et participantes}

Les procédures d'échantillonnage tiennent compte des objectifs de la recherche et du cadre théorique retenus. Nous avons choisi intentionnellement un site composé de 84 ménages d'orphelins rescapés du génocide. Ce site est composé de 400 orphelins regroupés dans une association nommée « Tubeho ». Suite à l'accord préalable des membres de l'association pour participer au projet, nous avons identifié les orphelins qui remplissaient les critères de sélection suivants: être orphelin rescapé du génocide, vivre dans un ménage d'orphelins, être aux études supérieures ou les avoir déjà complétées. Ensuite, nous avons rencontré chacune et chacun d'entre eux pour leur présenter le projet et les objectifs visés, et solliciter leur participation. Treize orphelins sur les 25 qui remplissaient les critères ont accepté de participer au projet. Ces jeunes étaient âgés entre 20 et 29 ans, signifiant qu'au moment du génocide, ils avaient entre 9 et 18 ans. Le tableau 1 présente les caractéristiques de l'échantillon.

\section{Procédures}

Pour recueillir les données, des entretiens individuels semi-dirigés ont été menés sur place. Au cours de ces entretiens, les participants et participantes avaient le droit de ne pas répondre aux questions ou de se retirer de la recherche. Un journal de bord a également été tenu et a servi de complément aux entretiens au moment du traitement et de l'analyse des données.

\section{Analyse des données}

En ce qui a trait au traitement des données, une lecture flottante a été réalisée afin de dégager les lignes directrices de chacun des entretiens individuels, puis de l'ensemble de ces entretiens. Le codage effectué à l'aide du logiciel NVivo a permis de repérer, de classer, d'ordonner et de condenser les unités de sens contenus dans les entretiens sous différentes catégories selon la démarche préconisée par Van der Maren (1996). La catégorisation s'est avérée essentielle car une catégorie tient à tout un ensemble d'autres catégories et met en marche l'articulation du sens des représentations, des vécus et des événements consignés (Paillé \& Muchielli, 2003), ce qui correspondait aux données de notre enquête de terrain. 


\section{Tableau 1}

Présentation des participants et participantes

\begin{tabular}{|c|c|c|c|c|c|c|}
\hline $\begin{array}{l}\text { Partici- } \\
\operatorname{pant}(\mathrm{e})\end{array}$ & Sexe & $\hat{A} g e$ & Scolarité & $\begin{array}{c}\text { Chef de ménage } \\
\text { ou non }\end{array}$ & Scolarité de la mère & Scolarité du père \\
\hline E 01 & $\mathrm{~F}$ & 28 & à l'université & oui & - & - \\
\hline E 02 & $\mathrm{~F}$ & 25 & à l'université & non & - & 5 ans secondaire \\
\hline E 03 & M & 29 & à l'université & oui & - & 4 ans secondaire \\
\hline Е 04 & $\mathrm{~F}$ & 28 & à l'université & non & métier & secondaire \\
\hline E05 & $\mathrm{F}$ & 22 & à l'université & oui & 4 ans secondaire & secondaire \\
\hline Е 06 & M & 29 & à l'université & oui & - & 5 ans secondaire \\
\hline Е 07 & $\mathrm{~F}$ & 29 & complété & oui & secondaire & secondaire \\
\hline E 08 & $\mathrm{~F}$ & 26 & complété & oui & secondaire & maîtrise \\
\hline E 09 & $\mathrm{~F}$ & 27 & à l'université & non & - & secondaire \\
\hline E10 & M & 30 & complété & oui & secondaire & secondaire \\
\hline E11 & $\mathrm{F}$ & 23 & à l'université & oui & primaire & primaire \\
\hline E12 & $\mathrm{F}$ & 23 & à l'université & non & secondaire & secondaire \\
\hline E13 & M & 20 & à l'université & non & primaire & 5 ans secondaire \\
\hline
\end{tabular}

-: n'ayant pas fait d'études

E: entretien

F: féminin

M: masculin

complété: ayant complété les études universitaires (baccalauréat ou licence)

\section{RÉSULTATS}

Les résultats de cette étude se regroupent en quatre grandes catégories déterminées a priori. La considération de la scolarité des participants et participantes avant le génocide nécessitait qu'on tienne compte de la famille en ce qui concerne la scolarisation des enfants. Cette catégorie réunit donc les propos recueillis au regard de l'importance que les parents accordaient aux études, du soutien qu'ils offraient à leurs enfants sur le plan scolaire et de leurs réactions face à l'échec ou aux difficultés scolaires de leurs enfants. La deuxième catégorie renvoie aux caractéristiques personnelles des participants et participantes: à leur intérêt par rapport aux études, aux difficultés qu'ils ont rencontrées et qui ont affecté leur scolarité ainsi qu'aux moyens qui leur ont permis de les surmonter et de concilier les responsabilités familiales et les tâches scolaires. La troisième catégorie réfère au soutien que les participants et participantes ont reçu du milieu scolaire, notamment des responsables de l'école, des enseignants et enseignantes et des pairs. La dernière catégorie a trait à la contribution de la communauté sur le plan scolaire. La résilience pour cette population est le produit de l'interaction de l'ensemble des ressources en provenance de toutes ces catégories.

\section{Soutien du milieu familial}

D'après ce qui se dégage des résultats, avant le génocide, les participants et participantes indiquent avoir reçu, de la part de leurs parents, l'affection et le soutien nécessaires sur le plan scolaire. Les 
parents, et plus particulièrement les pères, n'avaient pas seulement inscrit leurs enfants à l'école, mais ils leur ont fourni le matériel requis et les ont encouragés à étudier et à réussir par des conseils verbaux, des exemples, des récompenses lors de la réussite et des punitions quand ils échouaient. Ces derniers ont aussi fait de leur mieux pour protéger leurs enfants contre la discrimination à caractère ethnique qu'ils vivaient dans le milieu scolaire. Cela est souligné par le fait que les parents cherchaient des écoles privées quand leurs enfants ne pouvaient pas accéder aux écoles de l'État à cause de cette discrimination. L'importance que les parents des participants et participantes accordaient aux études était beaucoup liée à la perspective d'un meilleur avenir qu'ils préconisaient pour leurs enfants. Les propos des participants et participantes montrent la portée de ce soutien:

Ils accordaient une grande importance à l'école, car ils faisaient de leur mieux pour que chaque enfant aille à l'école à tout prix. (32: E1)

Nos parents nous encourageaient à étudier, de telle sorte qu'ils nous disputaient sévèrement quand ils remarquaient que nous n'étudiions pas bien. (289: E3)

Un autre élément important qui a soutenu la résilience des étudiantes et étudiants rescapés du génocide qui vivent seuls dans les ménages est la façon dont leurs parents se comportaient dans les moments difficiles. Le courage et la patience qu'ils ont manifestés ont servi de modèle à leurs enfants après le génocide, comme dans l'exemple de la participante qui compare ses souffrances d'après le génocide à celles que son père a vécu avant le génocide:

Mon père a été fortement battu, de telle sorte que même sa mort, c'était comme un repos pour lui. Moi, personne ne me battait, j'étais battue indirectement, car j'étais battue moralement. Mais, lui était très courageux, je me demandais alors pourquoi je ne pouvais pas rester courageuse. (1174: E8)

En résumé, tous les participants et participantes ont eu des bases solides de la part de leurs familles en matière de soutien à la poursuite des études avant le drame. Les parents des participants et participantes à la présente recherche ont été des véritables tuteurs de résilience pour leurs enfants.

\section{Caractéristiques individuelles}

Cette catégorie regroupe les propos reliés aux difficultés que les participants et participantes ont rencontrées, leur intérêt à poursuivre les études et les moyens qui leur ont permis d'y arriver. Après le génocide, tous les participants et participantes à la recherche se sont heurtés aux difficultés liées à la survie et ce avec un traumatisme élevé, car beaucoup d'entre eux venaient de côtoyer la mort. Par exemple, une participante a mentionné avoir reçu des coups de machette sur la tête pendant le génocide, ce qui a nui par la suite à sa concentration dans ses études. Elle décrit cet état comme suit:

J'avais un problème des maux de tête, à cause des coups de machette que j'ai eus pendant le génocide. Quand je faisais ma $4^{\mathrm{e}}$ année, j'ai été gravement malade. Si je n'avais pas cette détermination de poursuivre mes études, j'aurais pu abandonner. (1439: E9)

De plus, les participants et participantes n'avaient plus accès à leur foyer d'avant le génocide. Certaines et certains se sont retrouvés dans des orphelinats (E5, E8), d'autres dans des familles d'accueil qui pouvaient être ou non de leur parenté (E2, E9, E11, E12, E13) et d'autres seuls dans les ménages d'orphelins (E1, E3, E4, E6, E7, E10). Dans l'une ou l'autre de ces situations, les conditions de vie 
étaient difficiles. Avec la politique gouvernementale, celles et ceux qui étaient dans des orphelinats ont été reçus dans des familles. Quelques familles ont accueilli les enfants pour profiter des biens qui appartenaient à leurs familles disparues. C'est le cas d'une participante qui a passé des années en justice contre la famille d'accueil qui venait de s'approprier les terrains de ses parents. Dans d'autres familles, les orphelins ont été maltraités ou exploités pour accomplir des travaux domestiques (E5, E8). Voici un exemple de l'expérience vécue dans les familles d'accueil:

Tu comprends que je ne pouvais pas bien étudier. Nous étions maltraités. Vivre dans une famille maltraitante, c'est difficile [...] C'était une grande parcelle bien située. Cette famille voulait nous la prendre. Pour aller en justice, il me fallait trouver un endroit où mettre mes frères et sœurs en vue de les épargner. Par chance il y a eu une tante, qui a accepté de les héberger. Et j'ai pu aller en justice contre cette famille. (1161: E8)

Dans les ménages où vivaient presque la moitié des participants et participantes depuis la fin du génocide et où les autres les ont rejoints après le passage dans des orphelinats et dans des familles d'accueil, les orphelins sont devenus responsables d'eux-mêmes et ont dû assumer le rôle parental auprès des autres membres de la fratrie. Ces obligations représentaient un autre type de difficultés:

J'étudiais avec beaucoup de difficultés, dont le fait que j'étais responsable du ménage, mes résultats scolaires ont baissé par rapport à ceux que j'avais avant le génocide. (013: E7)

Je rencontre souvent des problèmes, surtout liés au soutien de mes frères et sœurs, ce sont des enfants en bas âge. Leur trouver le matériel scolaire, les nourrir. (1663: E11)

Vivre dans de telles conditions et aller de l'avant sur le plan scolaire requiert certainement un certain intérêt pour les études. Les entretiens démontrent que le retour à l'école s'est produit suite à la prise de conscience du fait qu'il leur revenait de se forger un avenir. Dans cette perspective, l'école se trouvait mieux placée. Les études leur permettraient de devenir autonomes, de se prendre en charge, de mieux assumer leurs responsabilités à l'endroit de ceux et celles qui sont à leur charge, de se développer et de développer leur pays, sans oublier pour autant la question de la promotion sociale et de revanche contre ceux qui ont voulu les anéantir. Bref, c'est surtout la projection dans l'avenir relative à ces différents aspects qui est la source principale de leur intérêt pour les études. Voici comment les participants et participantes expriment leur intérêt pour les études:

Je me rappelle, quand les écoles ont ouvert les portes après le génocide, je me suis assise et j'ai pensé, j'ai pris conscience qu'il n'y a pas d'autre vie à attendre, c'était difficile de vivre tel que nous vivions, et j'ai choisi de retourner à l'école. (417: E4)

Mon intérêt pour les études vient du fait que je puisse être utile pour mes frères et sœurs et pour développer mon pays. (2023: E13)

J'ai lutté pour que ceux qui ont tué mes parents, mes frères et sœurs ne me voient jamais dans les mauvaises conditions. (50: E1)

Avoir de l'intérêt et de la motivation ne suffit pas pour surmonter les difficultés auxquelles les interviewées et interviewés étaient confrontés. Il fallait d'autres stratégies pour atteindre leurs objectifs. Les plus chanceux avaient de la facilité dans les études, et même s'ils ne pouvaient pas exceller, ils avaient les résultats requis pour être promus d'une année à l'autre. Ensuite, il y a ceux et celles qui attestent que la foi à travers la prière leur a été un support inestimable. Les autres mentionnent le rôle 
joué par leurs compétences relationnelles, leur forte personnalité, leurs capacités de débrouillardise ainsi que le fait de voir autour d'eux des personnes qui vivaient dans des conditions plus difficiles que les leurs. Grâce à ces caractéristiques, ils arrivaient à concilier la vie dans les ménages avec les études. En voici quelques extraits:

Je prie et j'adresse à Dieu tous mes problèmes et après je me sens bien. (95: E2)

J'avais beaucoup de facilité pour les études, car si ça n'avait pas été cela, j'aurais échoué. De toute façon, je ne révisais pas mes cours. Je rentrais de l'école et immédiatement, j'entrais dans les tâches ménagères pour m'occuper de mes frères et sœurs. (1021: E7)

J'avais beaucoup de consolation par rapport aux autres enfants de mon âge que je voyais dans mon entourage et qui souffraient plus que moi, je devenais forte. (175: E8)

Ce qui m'a aidé beaucoup, c'est avoir une personnalité forte. (1269: E8)

\section{Soutien du milieu scolaire}

Cette section discute le soutien apporté par le milieu scolaire. Tous parlent d'une rencontre ou de plusieurs rencontres avec des acteurs de ce milieu qui ont contribué à leur résilience. Ces individus rencontrés allaient des responsables des établissements scolaires aux collègues de classe, tel que souligné dans les extraits suivants:

La personne qui m'a beaucoup marqué, c'est ce directeur qui m'a accueilli dans son école la première fois après le génocide. Il m'a accueilli dans une école privée sans savoir où j'allais trouver l'argent pour couvrir les frais scolaires. (1419: E9)

Mon enseignante de $5^{\mathrm{e}}$ et de $6^{\mathrm{e}}$ primaire m'a beaucoup aidé par sa compréhension. Je pouvais lui expliquer facilement les raisons de mon retard, les difficultés de me concentrer à l'école alors que j'avais d'autres charges à la maison qui me fatiguaient et qui n'étaient pas proportionnelles à mes forces et à mon âge. (682: E5)

Nous avons créé les associations des élèves rescapés; il s'agissait de forums qui nous permettaient de discuter de nos difficultés et de se projeter dans l'avenir [...] nous nous aidions mutuellement. (331: E3)

Le soutien et les interactions du milieu scolaire sont donc venus contrebalancer les difficultés de la vie ordinaire dans les ménages d'orphelins.

\section{Soutien de la communauté}

Cette partie englobe l'aide provenant des anciens amis et amies des familles des participants et participantes, des rencontres dues la plupart du temps par hasard, ou encore de l'État et des organismes locaux créés pour venir en aide aux rescapés du génocide. Le principal organisme est le Fonds national d'assistance aux rescapés du génocide (FARG) qui a payé les frais scolaires au secondaire pour les élèves rescapés du génocide et pour les études universitaires de ceux et celles qui remplissaient les critères d'excellence exigés par le FARG. Ce fonds a octroyé des logements aux participants et participantes de la présente recherche, diminuant ainsi les préoccupations reliées au problème d'habitation.

Un autre apport de l'État tient au fait d'avoir supprimé la discrimination à caractère ethnique dans les écoles. Comme certains participants et participantes l'ont mentionné au cours des entretiens, le 
système scolaire d'après le génocide a cessé de discriminer les élèves en se basant sur leur ethnie. Pour certains et certaines, le fait de ne plus être confrontés à cette discrimination leur a permis de poursuivre leurs études aisément. Un participant a même mentionné que le fait d'avoir un directeur Tutsi lui a permis de se confier à lui sans se poser des questions liées à la réception de ses confidences. Les associations qui sont venues en aide aux participants et participantes sont: l'Association des orphelins chefs de ménages (AOCM), l'Association des étudiants rescapés du génocide (AERG), Barakabaho et Kanyarwanda. Ces associations contribuaient à défrayer les coûts associés aux frais de scolarité, au matériel scolaire et parfois aux provisions alimentaires et à l'habillement. Voici quelques témoignages en rapport avec le soutien de la communauté:

Le prêtre me donnait le matériel scolaire et payait les frais scolaires. (464: E4)

Il est clair qu'après le génocide, il y a eu la Croix-Rouge, la famille d'accueil, le Fonds national d'assistance aux rescapés du génocide (FARG) et l'Association des étudiants rescapés du génocide (AERG) pour le moment. (621: E5)

L'État quand il a donné des écoles, il n'a pas fait de distinction, en disant ceux-ci sont Tutsis, d'autres sont Hutus, [...] puis j'étais très content qu'on n'ait pas à différencier les Hutus et les Tutsis, comme c'était avant. (1264:E8)

\section{Synthèse}

D'après le parcours et les témoignages des participants et participantes, nous pouvons déduire que la résilience n'est pas le fruit d'un seul facteur. Elle résulte de l'interaction de plusieurs facteurs, les uns agissant au même moment que les autres ou après. Ces facteurs permettaient de maintenir l'équilibre des participants et participantes en atténuant l'impact des facteurs de risque auxquels ils faisaient et font toujours face. Cela leur a permis d'avancer plus ou moins normalement dans la vie, malgré les difficultés qu'ils ont vécues et que certaines et certains d'entre eux vivent encore aujourd'hui. Le tableau 2 présente une synthèse des résultats de l'étude.

La figure 1 propose une modélisation du processus de résilience élaborée à partir des résultats de la recherche. En premier lieu, il y a les éléments de la famille, en particulier l'importance qu'ils accordaient à la scolarisation de leurs enfants, puis le soutien apporté dans ce contexte. Vient en troisième lieu le génocide qui vient changer le tournant de l'existence des participants et participantes. En quatrième et cinquième lieu viennent respectivement les éléments internes et externes qui ont soutenu la poursuite des études des participants et participantes après le génocide. Le dernier cadre est le résultat de l'interaction des différents encadrés précédents à l'exception du troisième. Il faut noter cependant qu'il y a des moments ou chaque élément peut intervenir seul dans le processus de résilience. En clair, ces éléments peuvent agir seuls ou se compléter les uns aux autres ou agir en interaction.

\section{DISCUSSION}

Au terme de la présente recherche, il apparaît évident que les participants et participantes font preuve de résilience, du moins tel que vu de l'extérieur. Toutefois, il est à noter que nous ne savons pas grand-chose sur leur monde intérieur, c'est-à-dire sur leurs séquelles psychologiques. Nous nous limitons à considérer la conciliation de leurs études à la vie dans les ménages comme un processus de résilience 
REVUE CANADIENNE DE SANTÉ MENTALE COMMUNAUTAIRE

\section{Tableau 2}

Présentation des résultats: catégories, codes et sous-codes retenus

\begin{tabular}{|c|c|c|c|}
\hline Catégories & Codes & Sous-codes & Nombre de participant(e)s* \\
\hline \multirow[t]{9}{*}{ Soutien familial } & Importance & & 13 \\
\hline & Soutien aux études & Matériellement & 13 \\
\hline & & Suivi & 12 \\
\hline & & Récompense & 4 \\
\hline & & Conseil & 4 \\
\hline & & Affectif & 3 \\
\hline & Réaction à l'échec & Punition & 7 \\
\hline & & Encouragements et aide & 6 \\
\hline & & Dialogue & 4 \\
\hline \multirow{33}{*}{$\begin{array}{l}\text { Caractéristiques } \\
\text { personnelles }\end{array}$} & Intérêt & Préparation de l'avenir & 12 \\
\hline & & Emploi & 9 \\
\hline & & Responsabilité & 8 \\
\hline & & Développement & 8 \\
\hline & & Instruction & 6 \\
\hline & & Revanche & 4 \\
\hline & & Promotion sociale & 4 \\
\hline & & Éducation parentale & 3 \\
\hline & & Culture & 1 \\
\hline & Difficultés & Matériel & 12 \\
\hline & & Psychologique & 9 \\
\hline & & Trop de responsabilités & 9 \\
\hline & & Maltraitance & 4 \\
\hline & & Ségrégation & 3 \\
\hline & Moyen & Prise de conscience & 11 \\
\hline & & Débrouillardise & 7 \\
\hline & & Appel à des connaissances & 6 \\
\hline & & Jobs & 5 \\
\hline & & Foi & 4 \\
\hline & & Sociabilité & 4 \\
\hline & & $\begin{array}{l}\text { Suivre des conseils et } \\
\text { exemples des parents }\end{array}$ & 4 \\
\hline & & Facilité dans les études & 4 \\
\hline & & Comparaison & 4 \\
\hline & & Persévérance & 3 \\
\hline & & Sens des responsabilités & 2 \\
\hline & & Appel à la parenté & 2 \\
\hline & & Appel à des amis & 2 \\
\hline & & Sentiment d'autonomie & 2 \\
\hline & & Patrimoine familial & 2 \\
\hline & & Volonté & 2 \\
\hline & & Culture & 1 \\
\hline & & Loisir & 1 \\
\hline & & Oubli & 1 \\
\hline
\end{tabular}




\section{Tableau 2}

(suite)

\begin{tabular}{lllc}
\hline Catégories & Codes & Sous-codes & Nombre de participant(e)s* \\
\hline Soutien de & Soutien du milieu & Responsables des établissements & 13 \\
l'environnement & scolaire & Relation enseignant(e)-élève & 10 \\
& & Apport des ami(e)s & 10 \\
& & Soutien entre victimes & 9 \\
& Soutien de la & État & 13 \\
& communauté & Organismes & 12 \\
\hline
\end{tabular}

* Nombre de participants et participantes ayant donné comme réponse cette unité de sens

que nous traitons sous l'angle de l'équilibre. Cyrulnik (1999) souligne que presque tous les enfants résilients ont eu à répondre à une première question: « Pourquoi dois-je tant souffrir? » Et cette question les a incités à réfléchir comme suit: « Comment vais-je faire pour être heureux quand même? » (p. 15).

Dans notre recherche, les mêmes questions se posent implicitement, car pour sortir de leur situation difficile, les orphelins ont d'abord pris conscience de leur situation problématique et ont opté pour la poursuite des études comme voie de solution. Pour eux, la réussite scolaire leur garantissait dans une certaine mesure une porte de sortie vers un avenir meilleur. Parmi les traits personnels des participants et participantes qui font partie des caractéristiques des résilients et résilientes figurent l'autonomie, le sens des responsabilités et de l'engagement, l'altruisme, la foi, la capacité de résoudre des problèmes, la débrouillardise, la comparaison avec d'autres situations plus difficiles qui permettaient de relativiser leurs propres problèmes et les compétences sociales (Benard, 2004; Vanistendael \& Lecomte, 2000).

L'idée de revanche est aussi apparue parmi les éléments qui ont favorisé le processus de résilience. La revanche chez les orphelins rescapés du génocide est dirigée vers ceux qui ont tué leurs parents. Ils ne souhaitent pas que ces derniers les voient dans des conditions malheureuses, car ce serait leur avouer qu'ils ont gagné. Cette revanche est aussi une façon de représenter celles et ceux qui sont partis, d'honorer leur mémoire et de leur rester fidèle.

Cependant, il est important de mentionner que, bien que cette fidélité et cette honnêteté envers les leurs aient contribué à leur processus de résilience, elles peuvent comporter un courage pathologique. Ce constat est souligné par Cyrulnik (2004) qui avance que certains orphelins de guerre qui avaient bénéficié d'un attachement sécure avec leurs parents ont continué à s'y accrocher même après la disparition de ces derniers. L'auteur indique que le lien d'attachement qu'ils ont tissé restait parfait, étant donné que les parents morts ne pouvaient plus commettre d'erreur éducative. Cyrulnik (2004) explique également que ces orphelins restent encore plus fidèles aux vœux de leurs parents défunts. Cela pourrait fort bien s'appliquer à quelques-uns de nos participants et participantes. Toutefois, c'est un aspect qui n'a pas été approfondi, le seul constat étant qu'avant leur départ, les parents leur avaient donné des bases solides autant sur le plan affectif que sur le plan de la motivation scolaire. Les parents ont motivé leurs enfants par l'importance qu'ils accordaient aux études et le soutien qu'ils leur ont 


\section{Figure 1}

Modélisation du processus de résilience chez les étudiant(e)s orphelins rescapés du génocide qui vivent seuls dans les ménages au Rwanda

1. Importance accordée aux études par les parents avant le génocide:

- un meilleur avenir

- auto-prise en charge

5. Ressources externes après le génocide:

- parenté

- ami(e)s des parents

- rencontres

- responsables d'école

- enseignant(e)s

- ami(e)s et collègues de classe

- victimes

- associations de rescapés

- associations d'aide aux

vulnérables

- État

- absence de discrimination

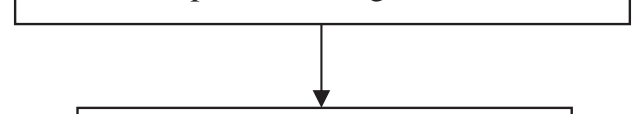

2. Soutien familial:

- encouragements

- conseils

- soutien matériel et affectif

- récompense

- suivi

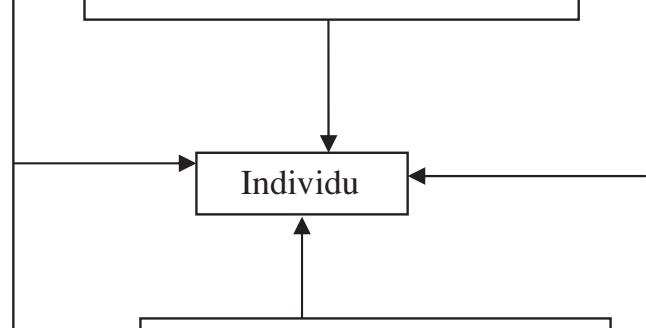

4. Ressources internes après le génocide:

- prise de conscience de la réalité

- résolution de problèmes

- initiative

- prise de décision

- détermination

- autonomie

- revanche

- fidélité aux parents morts

- foi

6. Résilience
3. Pendant le génocide:

- perte des parents

- confrontation à la mort

- bouleversement

- traumatisme

- responsabilité

- pauvreté

- maltraitance 
octroyé avant de mourir. Restés seuls, les enfants se sont appuyés sur ce bagage considéré comme un héritage pour faire face à « l'après génocide».

Le fait de devoir compter sur eux-mêmes et de devenir responsables des frères et sœurs est devenu le centre de la nouvelle vie des orphelins qui vivent seuls dans les ménages. En d'autres termes, soutenir les autres membres de la fratrie est devenu une mission, une raison de vivre débout. Or, d'après Cyrulnik (2004) et Lecomte (2004), la recherche de sens est l'un des éléments essentiels de la résilience. Ensuite, comme on ne peut être résilient seul (Lecomte, 2004), la création des liens est un autre élément crucial qui soutient la résilience (Anaut, 2003; Benard, 2004; Bouteyre, 2004; Lecomte, 2004; Poletti \& Dobbs, 2001). Ce constat rejoint les résultats de cette étude, car tout au long de la trajectoire des orphelins, à l'école et à l'extérieur de ce milieu, il y a eu toujours quelqu'un ou quelqu'une (parenté, directeur ou directrice d'école, enseignante ou enseignant, autre adulte rencontre par hasard, collègue de classe) pour leur tendre la main et marcher avec eux sur la route de la survie.

Un autre élément considéré comme un tremplin vers la résilience est la reconnaissance que la société et la culture accordent à la blessure et à la blessée ou au blessé et les moyens mis en place pour aider celle-ci ou celui-ci (Cyrulnik, 2004; Ehrensaft \& Tousignant, 2001; Lecomte, 2004). Dans l'étude actuelle, la création par l'État du Fonds national d'assistance aux rescapés du génocide constitue un acte de reconnaissance important. De plus, la mise en place des différentes associations de rescapés du génocide créées progressivement (Ibuka, Avega, AOCM, Barakabaho, AERG) pour mobiliser les mécanismes de soutien et d'aide aux rescapées et rescapés leur a donné la possibilité d'être représentés et donc d'exister. Il reste à veiller à ce que ces organismes d'aide ne maintiennent pas dans le statut de vulnérabilité ceux et celles qu'ils soutiennent, car cela empêcherait le développement de la résilience (Cyrulnik, 2004) à leur insu.

\section{CONCLUSION}

La présente recherche a permis de dégager les facteurs qui ont contribué à la résilience des orphelins rescapés du génocide qui vivent seuls dans les ménages au Rwanda. L’interaction de ces divers facteurs (individuels, familiaux, du milieu scolaire et de la communauté) a soutenu le processus de résilience des participants et participantes. Nous ne pouvons pas prétendre avoir fait un inventaire exhaustif de tous les facteurs qui accompagnent les orphelins rescapés du génocide qui vivent seuls dans les ménages au Rwanda sur la voie de la résilience. Toutefois, les éléments mis en évidence dans le cadre de la présente étude peuvent servir de base à un programme d'éducation destiné à promouvoir la résilience auprès de différentes populations de la communauté rwandaise affecté par le génocide des Tutsis.

D'autres aspects de cette recherche n'ont pas été suffisamment approfondis, faute de temps et de moyens dont la chercheure ne disposait pas au moment de la recherche. Il est également important de noter que les résultats de cette étude ne sont pas généralisables à toute la population des étudiantes et étudiants rescapés. Ils sont applicables à celles et ceux qui ont participé à l'étude, étant donné que l'expérience de chacun(e) est unique, même si les facteurs qui les ont soutenus semblent analogues. Cette analogie permettrait si nécessaire de transférer ces résultats dans des contextes similaires. 


\title{
REVUE CANADIENNE DE SANTÉ MENTALE COMMUNAUTAIRE
}

\begin{abstract}
This article reports the main results of a study carried out among orphans who survived the 1994 genocide of Tutsis in Rwanda and live on their own or head households. The goal of the study was to understand the protective factors that helped some of them survive in their households while at the same time pursuing their studies as far as university, despite being faced with multiple risk factors during and after the genocide. These protective factors are related to individual characteristics as well as family and community. The article briefly outlines the context of the study, the method used and the results that were obtained, and presents recommendations for future studies on resilience in the Rwandan community and in other similar contexts.
\end{abstract}

\section{RÉFÉRENCES}

Anaut, M. (2003). La résilience: surmonter les traumatismes. Paris: Nathan.

Association de coopération et de recherche pour le développement (ACORD). (2001). La situation des enfants chefs de ménage au Rwanda: un défi de taille. Kigali, Rwanda: Auteur.

Bazigaba, J.C. (2005). Le vécu psychologique des enfants et des adolescents chefs de ménage orphelins du génocide: cas du village UYISENGA n'IMANZI de Kicukiro. Mémoire de licence inédit, Université nationale du Rwanda, Butare.

Benard, B. (2004). Resiliency: What we have learned. San Francisco: WestEd.

Bouteyre, S. (2004). Réussite et résilience scolaires: chez l'enfant de migrant. Paris: Dunod.

Cyrulnik, B. (1999). Un merveilleux malheur. Paris: Odile Jacob.

Cyrulnik, B. (2003). Échange avec des professionnels de la relation d'aide. Dans B. Cyrulnik \& C. Seron (dir.), La résilience ou comment renaître de sa souffrance (pp. 43-69). Paris: Fabert.

Cyrulnik, B. (2004). Parler d'amour au bord du gouffre. Paris: Odile Jacob.

Dushime, R. (2004). Étude des problèmes socio-économiques des enfants orphelins chefs de ménage au Rwanda après le génocide de 1994: cas du district de Nyamata. Mémoire de licence inédit, Université libre de Kigali, Kigali, Rwanda.

Ehrensaft, E., \& Tousignant, M. (2001). L'écologie humaine et sociale de la résilience. Dans M. Manciaux (dir.), La résilience: résister et se construire (pp. 125-136). Genève: Médecine \& Hygiène.

Gishoma, D. (2005). Analyse de la situation du traumatisme psychique dans les ménages dirigés par les enfants au Rwanda. Mémoire de licence inédit, Université nationale du Rwanda, Butare.

Lecomte, J. (2004). Guérir de son enfance. Paris: Odile Jacob.

Mukuzanyana, F. (2004). Situation sociale des enfants orphelins chefs de ménage au Rwanda: cas du district de Kanombe. Mémoire de licence inédit, Université libre de Kigali, Kigali, Rwanda.

Muragizi Kanama, A. (2004). Les principaux problèmes rencontrés par les veuves et les orphelins du génocide d'avril 1994: cas des membres d'Avega et d'AOCM résidents à Remera. Mémoire de licence inédit, Université libre de Kigali, Kigali, Rwanda.

Paillé, P., \& Muchielli, A. (2003). L'analyse qualitative en sciences humaines et sociales. Paris: Armand Colin. Poletti, R., \& Dobbs, B. (2001). La résilience: l'art de rebondir. Saint Julien-en-Genevois, France: Jouvence.

Royal, S. (2001). La lutte contre la maltraitance: un des chantiers majeurs du ministère de la famille et de l'enfance. Dans Fondation pour l'enfance (dir.), La résilience: le réalisme de l'espérance (pp. 13-23). Ramonville-Saint-Agne, France: Érès.

Rwabidadi, P. (2000). Problèmes socio-éducatifs des enfants chefs de famille orphelins du génocide d'avril 1994 au Rwanda: cas de la commune de Kicukiro. Mémoire de licence inédit, Université nationale du Rwanda, Butare.

St-Onge, M. (2003). Évaluation de l'impact d'une intervention précoce sur le développement global d'enfants préscolaires en lien avec les facteurs de risque et de protection. Mémoire de maîtrise inédit, Université du Québec à Trois-Rivières. 


\section{FACTEURS DE RÉSILIENCE CHEZ DES ORPHELINS RESCAPÉS DU GÉNOCIDE}

UNICEF. (2004). Dix ans après le génocide, les enfants rwandais supportent encore ses conséquences à long terme. Récupéré en ligne le 21 mars 2009 de: http://www.unicef.org/french/media/media_20325.html

Van der Maren, J.-M. (1996). Méthodes de recherche pour l'éducation. Montréal: Presses de l'Université de Montréal.

Vanistendael, S., \& Lecomte J. (2000). Le bonheur est toujours possible: construire la résilience. Paris: Bayard. 